九州大学学術情報リポジトリ

Kyushu University Institutional Repository

Short Crack Growth Behavior and its

Transitional Interaction with 3D Microstructure in $\mathrm{Ti}-6 \mathrm{Al}-4 \mathrm{~V}$

Hassanipour, M.

Department of Mechanical Engineering, Kyushu University

Watanabe, Satoshi

Department of Mechanical Engineering, Kyushu University

Hirayama, Kyosuke

Department of Mechanical Engineering, Kyushu University

Toda, Hiroyuki

Department of Mechanical Engineering, Kyushu University

他

ht tp://hdl. handle. net/2324/4123955

出版情報: Materials Science and Engineering：A. 738，pp.229-237，2018-12-19. Elsevier バージョン：

権利関係 : 


\title{
Short Crack Growth Behavior and its Transitional Interaction with 3D
}

\section{Microstructure in Ti-6Al-4V}

\author{
M. Hassanipour ${ }^{\text {a }}$, S. Watanabe ${ }^{\text {a }}$, K. Hirayama ${ }^{\text {a }}$, H. Toda ${ }^{\text {a }}$, K. Uesugi ${ }^{b}$, A. Takeuchi ${ }^{b}$ \\ ${ }^{a}$ Department of Mechanical Engineering, Kyushu University, 744, Motooka, Nishi Ward, Fukuoka, Fukuoka 819-0395, \\ Japan \\ ${ }^{\mathrm{b}}$ Japan Synchrotron Radiation Research Institute, 1-1-1, Kouto, Sayo Cho, Sayo Gun, Hyogo 679-5148, Japan
}

\begin{abstract}
Currently, short crack growth behavior and rate variations are not well understood in the literature. This is due to lack of studies regarding the interaction between 3D short crack and microstructure and its effect on crack growth. In order to study this interaction, in situ computed tomography was performed to measure crack growth at sub-grain level (every $5 \mu \mathrm{m}$ ) during a fatigue test in a bimodal Ti-6Al-4V alloy for two crack front regions. This was followed by serial sectioning coupled with electron backscattering diffraction (EBSD) to identify the short crack growth in the microstructure, i.e. $\alpha, \alpha+\beta$ phases and interface. Results show that crack growth has the highest rate in $\alpha$ phase as compared to the $\alpha+\beta$ phase and the interface in both regions. The crack grows preferably into $\alpha$ phase when compared to the average microstructural fraction in the first region, but it decreases below this fraction in the second region. The crack grows mainly close to crystallographic planes in $\alpha$ grains with the maximum shear stress (favorable planes) in the first region. As the short crack grows into the second region, there is an increase in number of grains enclosed in the plastic zone size. As a result, there is a decrease in the mismatch angle between neighboring cracked grains, which leads to higher deviation from favorable planes causing a local variation in crack growth rate.
\end{abstract}

Key Words: Short crack propagation, Ti-6Al-4V, Synchrotron radiation, Tomography, Crystallographic orientations, Electron backscatter diffraction

\section{Nomenclature}

\begin{tabular}{|c|c|c|c|}
\hline ND & normal direction & D & distance along crack front line at the last cycle \\
\hline TD & transverse direction & $\Delta \mathbf{a}$ & crack length \\
\hline RD & rolling direction & $\Delta \theta$ & image slice angle of crack in xy plane \\
\hline$\alpha$ & grains with primary alpha phase & $\boldsymbol{\alpha}+\boldsymbol{\beta}$ & grains with alpha and beta phase \\
\hline$\alpha / \alpha+\beta$ & interface between $\alpha$ and $\alpha+\beta$ & $\mathrm{da} / \mathrm{dN}$ & crack growth rate \\
\hline CRSS & $\begin{array}{l}\text { critical resolved shear stress } \\
\text { with the highest Schmid factor }\end{array}$ & 甲ave & $\begin{array}{l}\text { average crack plane mismatch angle with the } \\
\text { neighbouring cracked grains }\end{array}$ \\
\hline $\operatorname{Sch}_{\max }$ & Maximum Schmid factor & $\Psi$ & $\begin{array}{l}\text { deviation from crystallographic plane with the } \\
\text { highest Schmid factor }\end{array}$ \\
\hline
\end{tabular}

\section{Introduction}

Small defects are inevitably formed during the fabrication process in many engineering components such as aircraft blisks made of Ti-6Al-4V. As the cyclic loads are applied, these small defects or cracks start to propagate with high variations in growth rates. These variations are caused by the three dimensional (3D) variation in the microstructural features, i.e. crystallographic orientations, and the morphology of the grains on the surface and inside the material $[1,2]$. 
Since the microstructural parameters vary in each specimen and there is a lack of understanding of the 3D crack and grains interaction, multiple specimens are required in fatigue tests in order to have an upper and lower band for crack growth rate. Considering these bands, fatigue life is either overestimated or underestimated in the design, without detailed understanding of crack and grains interactions.

Crack formation and early (short) growth in the near-alpha titanium alloys is associated with the dislocation pile up along the high density slip planes in the $\alpha$ grains that are basal, prismatic and pyramidal in titanium alloys [3]. It is reported that the critical resolved shear stress (CRSS) on prismatic plane is slightly lower than the basal one, and three times lower than the pyramidal one in a single crystal of Ti-6Al-4V alloy [4-6]. However, these analysis conducted by transmission electron microscopy (TEM) were limited to single crystals and very local areas in polycrystalline titanium alloys.

In order to analyze a wider area, some studies have used 2D and 3D electron backscattered diffraction (EBSD) analysis in order to investigate the slip planes activation and cracks formation leading to fracture in polycrystalline Ti-6Al-4V [7,8]. It was observed that there is a lower number of grains with activated basal slip planes than the prismatic ones. However, most of the cracks initiating from grains with relatively high basal Schmid factors resulted in specimens fracture. It was concluded that a higher local concentration of these grains favors propagation leading to fracture $[9,10]$. It should be mentioned that the aforementioned studies were partly analyzed using 2D analysis which can results in some misleading interpretations. Moreover, these studies give a comparative and qualitative prediction that the aforementioned cluster of grains favors propagation without investigating other possible types of microstructural textures. For instance, other studies have shown that crack can initiate and lead to fracture through grains with high prismatic Schmid factors [11,12].

Early studies on the short crack propagation were mainly focused on the 2D surface [13-15]. The recent 3D analysis have used the global multiaxial fatigue failure criteria, such as the critical plane approach to predict the crack propagation stage in the local microstructure [16,17]. These failure criteria consider the combination of shear strain with normal stress on crack plane for each grain to propagate the crack front and to predict the fatigue failure $[18,19]$. However, interaction between crack and grains affects the crack plane orientation and its morphology leading to growth rate variations. For instance, it was reported that crack can grow through basal plane in grains with low plasticity (Schmid factors) in order to follow the crack planes in the neighboring cracked grains [20]. Other studies have shown that crack plane grows in a facet-like path with 5 to 20 degrees anomalous deviation from the basal plane in most of the grains in cyclic fatigue tests [2,9,21]. It was shown that a sharp decrease in the prismatic Schmid factor of the neighboring grains leads to crack arrest [20]. Other studies have shown that a change in grains crystallographic orientation along the crack plane induces tilt and twist causing a decrease in crack growth rate [22,23]. Thus, it can be concluded that the interaction with neighboring grains affect the crack plane orientation and leads to an increase or decrease of its growth rate till reaching the final fracture.

It was reported that the crack growth rate is higher in facet-like planes as compared to the non-facet ones at small crack length regions [1]. As the crack length becomes longer, the growth rate in facet and non-facet-like planes become close to each other. However, some studies have shown that crack with facet-like planes are not necessarily faster than non-facet ones [21,24]. This is due to the crack and grain transitional interaction, which depends on crack length and its driving forces which can be different in each alloy. Therefore, it can be said that the crack and grain interaction is transitional and length-dependent.

The 3D crack and grains interaction affects crack plane orientation and morphology in grains leading to variations in crack growth rate. In this study, the crack propagation is investigated at increasing number of cycles in the microstructure $(\alpha, \alpha+\beta$ phases, and interface) at sub-grain level growth $(5 \mu \mathrm{m})$. Moreover, as the short crack grows to become a longer, there will be a transition in the interaction between the 3D crack and microstructure. This transitional interaction and its effect on the 3D crack path and growth rate variations is studied. 


\section{Material and experimental procedures}

\subsection{Material}

The chemical composition of the Ti-6Al-4V alloy is given in Table 1 . This alloy was hot rolled at $800^{\circ} \mathrm{C}$ resulting a $50 \%$ overall thickness reduction. Afterwards, it was tempered up to $900^{\circ} \mathrm{C}$ during 96 hours in the furnace and cooled down to the room temperature. At the end of heat treatment, this alloy was composed of $65 \%$ hexagonal close-packed (hcp) primary $\alpha$ phase and $35 \%$ of acicular secondary $\alpha$ phase within the body center cubic (bcc) $\beta$ phase ( $\alpha+\beta$ phase) as shown in Figure 1 . The primary $\alpha$ average grain size diameter was equal to $20 \mu \mathrm{m}$. The ND, TD, and RD corresponds to normal, transverse and rolling directions, respectively. In the following sections ND, TD, and RD are referred as $\mathrm{x}, \mathrm{y}, \mathrm{z}$. Tensile test showed that ultimate tensile strength corresponds to $934 \mathrm{MPa}$.

Table 1. Chemical compositions of the studied material (mass \%).

\begin{tabular}{cccccccc}
\hline $\mathbf{T i}$ & $\mathbf{A l}$ & $\mathbf{V}$ & $\mathbf{C}$ & $\mathbf{H}$ & $\mathbf{F e}$ & $\mathbf{O}$ & $\mathbf{N}$ \\
\hline Bal. & $5.5 \sim 6.75$ & $3.5 \sim 4.5$ & 0.08 & 0.15 & 0.40 & 0.20 & 0.05
\end{tabular}

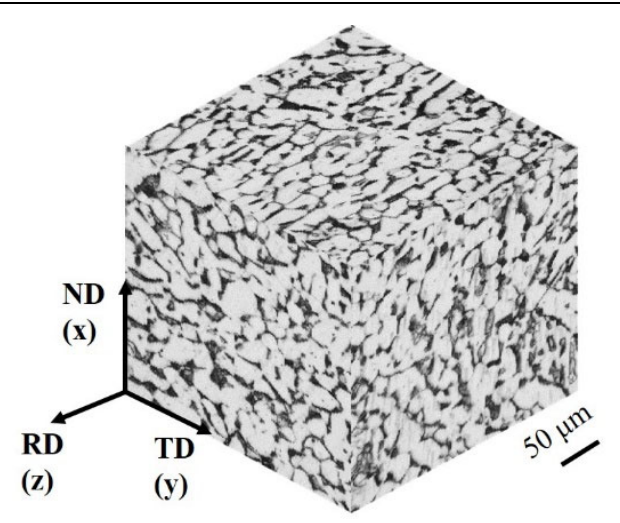

Figure 1. Revealed microstructure of the bimodal Ti-6Al-4V.

\subsection{Experimental procedure}

\subsubsection{Fatigue test using X-ray tomography}

A load-controlled fatigue test was performed at $\mathrm{R}=0.1$ with a frequency of $15 \mathrm{~Hz}$. A specimen with a square cross section of $800 \times 800 \mu \mathrm{m}$ was made by electro discharge machining. The gauge section was manually polish ed to obtain the $600 \times 600 \mu \mathrm{m}$ cross-section. This section was subjected to a maximum stress of $778 \mathrm{MPa}$. In order to be able to follow a main crack in the specimen a notch with dimensions of $60 \times 20 \times 4 \mu \mathrm{m}$ was made on the surface using a focused ion beam.

In situ computed tomography (CT) at the BL20XU beamline of SPring-8 was employed to measure scan the growing crack on the surface and inside the alloy. The monochromatic X-ray energy of $30 \mathrm{keV}$ was produced by a liquid nitrogen-cooled Si (111) double crystal monochromator. A CMOS camera (ORCA Flush 4.0, Hamamatsu Photonics K. K.) of 4.0 megapixels, with a $10 \mathrm{~mm}$ thick Lu2SiO5:Ce scintillator, was used for acquiring the projection images; and a $20 \times$ objective lens was used to obtain an effective pixel size of $6.5 \mu \mathrm{m} \times 6.5 \mu \mathrm{m}$. The sample distance to source and camera were $2400000 \mathrm{and} 53 \mathrm{~mm}$, respectively. During 180 degree of stage rotation with 0.1 degree step, 1800 scans were conducted with an exposure time of $400 \mathrm{~ms}[25]$.

Titanium alloy are prone to cold dwell effect which can be induced during the scanning. After some preliminary test, it was concluded that performing the scanning at $60 \%$ of maximum stress does not induce any cold dwell effect. The crack was periodically scanned for every $5 \mu \mathrm{m}$ of crack growth that is projected on the detector, for two different crack length regions in order to investigate a transitional interaction between crack and microstructure. The first region was between 105 and $156 \mu$ m, 
corresponding to 8700 and 10600 cycles, respectively. The second one was between 292 and $342 \mu \mathrm{m}$, corresponding to 17000 and 18560 cycles, respectively.

\subsubsection{Three dimensional imaging and crack front path in the microstructure}

After X-ray scanning, the image slices were reconstructed by using the conventional filtered back-projection algorithm. The linear absorption coefficient was converted to 16-bit grayscale from 0 to 63566 having voxels size equal to $0.515 \mu \mathrm{m}$. Afterwards, the absorption contrast images were normalized and a gray threshold was specified in order to generate the crack front area. The 3D crack front for each cycle was aligned in a 3D volume rendering software in order to compare precisely the crack front location in the alloy for different cycles.

The short crack front that grows without any interaction with the microstructure results in a homogenous quasi-semi elliptical shape for each cycle. However, the real short crack has a local interaction with the microstructure so its shape becomes irregular. As a result, in this study, we calculate the local crack growth rate along the crack front line for each cycle. The local crack length is defined as the distance between the notch and crack tip, which is $\Delta a$, at a defined angle of $\theta$ for three crack sections in Figure 2(a). As a result, the crack images were transferred from Cartesian coordinates $(x, y, z)$ to the polar coordinates $(r, \theta$, $z$ ) for section 1 and 3, (left and right side of the crack, respectively), which results in an image slice typically shown in Figure 2(b). Afterwards, the images were binarized and the crack tip coordinates were specified. Due to the value of gray threshold, crack length can be underestimated in some cycles leading to negative crack growth rates, however, without changing the threshold value the seed growth [26] for all slices were re-examined in order to have less than $5 \%$ error of this type in each cycle.

(a)

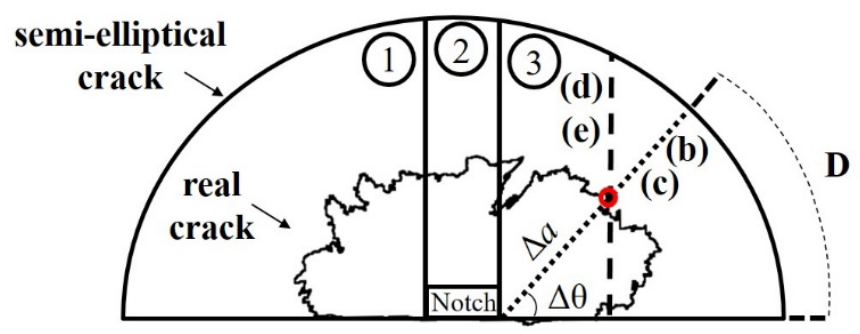

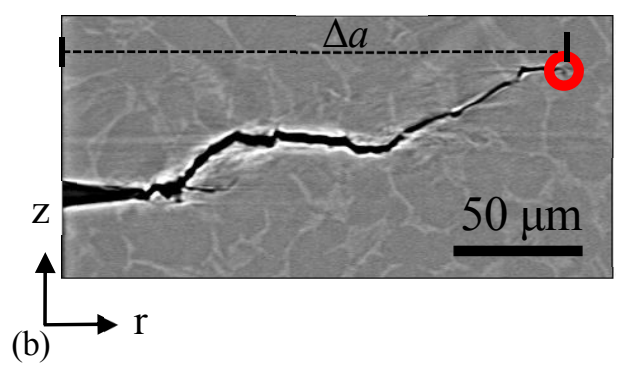

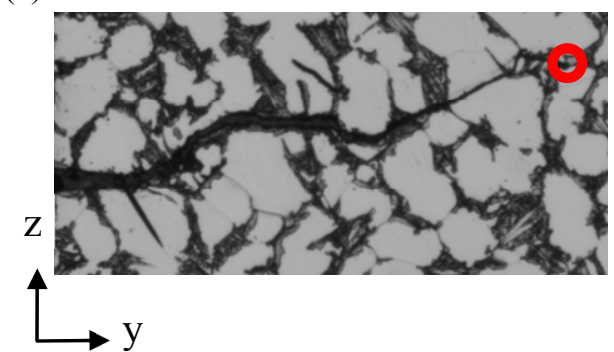

(d)

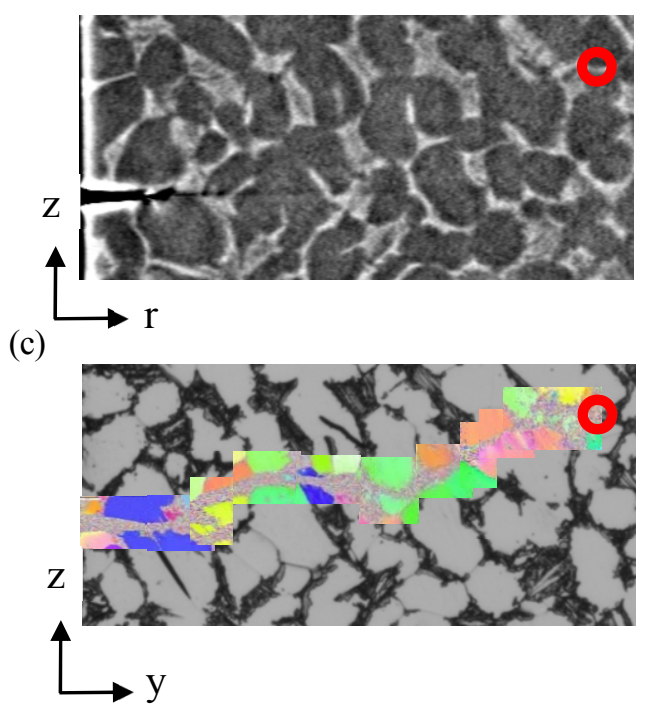

(e)

Figure 2. Schematic of (a) semi-elliptical and real crack with three different sections in order to determine the crack tip location in the microstructure by using, (b) absorption contrast, and (c) phase contrast image slices in polar coordinates for sections 1 and 3 and in Cartesian coordinates for section 2, followed by the serial sectioning procedure for grain identification using (d) etched, and (e) EBSD image slices in Cartesian coordinates. 
Defining a gray threshold value for $\alpha$ and $\alpha+\beta$ is difficult due to the dispersion of gray values in these phases. Therefore, a procedure was employed to make phase contrast images with a lower dispersions and higher difference between the gray values of the two phases (Figure 2(c)).

In order to generate the phase contrast images, the complex refractive index of X-ray, $n$, in titanium alloy was calculated. This index can be written as $n=1-\delta+i \beta$, where $\delta$ describes refraction or phase properties, and $\beta$ is related to the absorption coefficient. It is assumed that $\delta$ and $\beta$ are proportional to each other in this alloy which is suitable for light materials at high photon energies [27]. The values of $\delta$ and $\beta$ were calculated as described in [28] for the titanium alloy. Afterwards, propagation-based singledistance phase retrieval algorithm using Paganin's method was applied, to reconstruct the distribution of the complex X-ray refractive index from a single projection image per projection using the conventional filtered back projection algorithm [29].

This leads to images with a better distinction between the gray values of $\alpha$ and $\alpha+\beta$. Afterwards, images were binarized using an average gray threshold leading to black pixels as $\alpha$ phase and the white ones as $\alpha+\beta$ phase. A region of $3 \times 3 \times 3$ voxels, was defined around the crack tip, so 27 black voxels means that the crack tip is entirely in the $\alpha$ phase and no white voxels means that the crack tip is in the $\alpha+\beta$ phase, otherwise it will be the $\alpha / \alpha+\beta$ interface. The crack growing without any preference in the average microstructural fractions for each phase were calculated by considering the average of 5 semi-elliptical crack front lines in the microstructure.

\subsubsection{Three dimensional reconstruction of grains with $\alpha$ phase}

Afterwards, the specimen was polished through the thickness and EBSD was conducted around the crack path (Figure 2(d) and (e)). This serial sectioning procedure was carried out for 57 sections to identify the crystallographic orientations of grains in the $\alpha$ phase around the crack. The distance between each section was set by inducing a 3 micron indent in the specimen that was removed by polishing. Afterwards, EBSD was conducted around the crack path with a step size of $0.2 \mu \mathrm{m}$ followed by polishing and etching the next two sections in order to be able to follow the microstructure through thickness sections.

Afterwards using the EBSD and etched images, grains were identified on the absorption contrast images and they were employed to reconstruct the $3 \mathrm{D}$ grains. The crack front coordinates in different cycles that corresponds to each identified grain determines the crack growth rate in each grain. Using the crack front line coordinates in two subsequent cycles in each grain, two vectors were defined in order to calculate the crack plane normal in each grain. By using the 3D crack plane orientation within the grain and the 3D crystallographic grain orientation, i.e. Euler angles, the crack plane with respect to crystallographic planes were calculated.

\section{Results}

\subsection{Spatial and temporal crack growth rate variations in the microstructure}

The superimposed 3D crack front images for both crack front regions is shown in Figure 3. As it can be seen, in the first region (8700 to 10600 cycles) crack front has an irregular shape and it has lower growth on the right side near the specimen surface as compared to the left one. This implies that crack front has a tendency to grow in some location in the microstructure more than the others especially in the first region. However, this tendency becomes less significant in the second region. 


\section{Number of cycles in} the first region
- 8700
- 8900
- 9080
- 9250
- 9410
- 9710
- 9990
- 10200
- 10400
- 10600

in

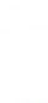

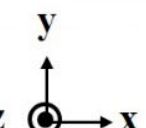

Number of cycles in the second region

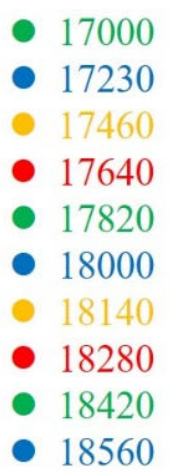

\section{Crack on the specimen surface}

Figure 3. Superimposed three dimensional crack front images projected on xy plane with three sections and different colors corresponding to the first region (from 8700 to 10600 cycles) followed by the second region (from 17000 to 18560 cycles).

Regarding the $\alpha$ phase, a total of 198 grains were identified, which consist of cracked grains and their surrounding grains. A number of 15 and 34 cracked grains were identified in the first and second region, respectively. Spatial crack growth rate variations in $\alpha, \alpha+\beta$ phase and interface $(\alpha / \alpha+\beta)$ in both regions are shown in Figure 4.

In the first region, the crack front growth rates are high on the left side (section 1) at 8900 cycles (Figure 4(a)), this is due to crack growth in facet-like planes in grains as compared to the non-facet-like ones. However, the crack growth rates on the right side become close to the left side at 10600 cycles, which shows the interaction between left and right side of the crack front (Figure 4(b)). In the second region, the crack front growth rate is similar along the crack front at 17230 cycles with increasing percentage of crack front in $\alpha+\beta$ phase and interface (Figure 4(c)). The crack growth rate increases in some $\alpha$ grains, in $\alpha+\beta$ phase and interface at the last cycle (Figure 4(d)).

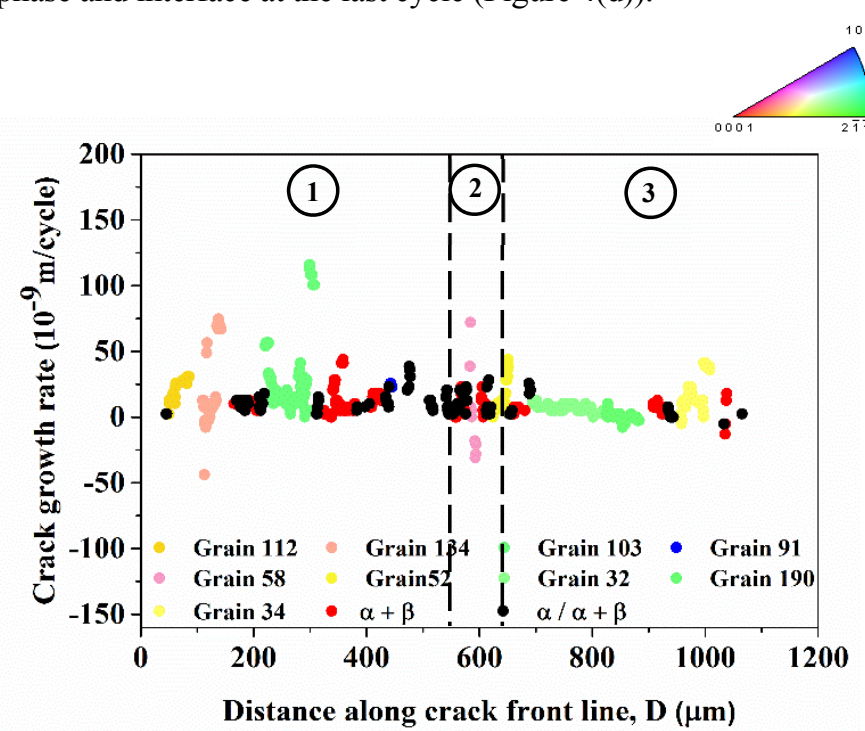

(a)

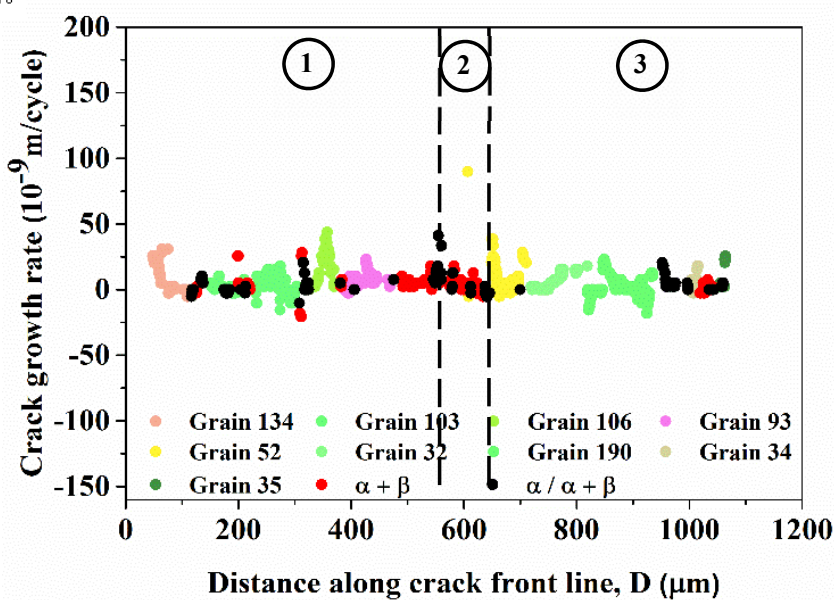

(b) 


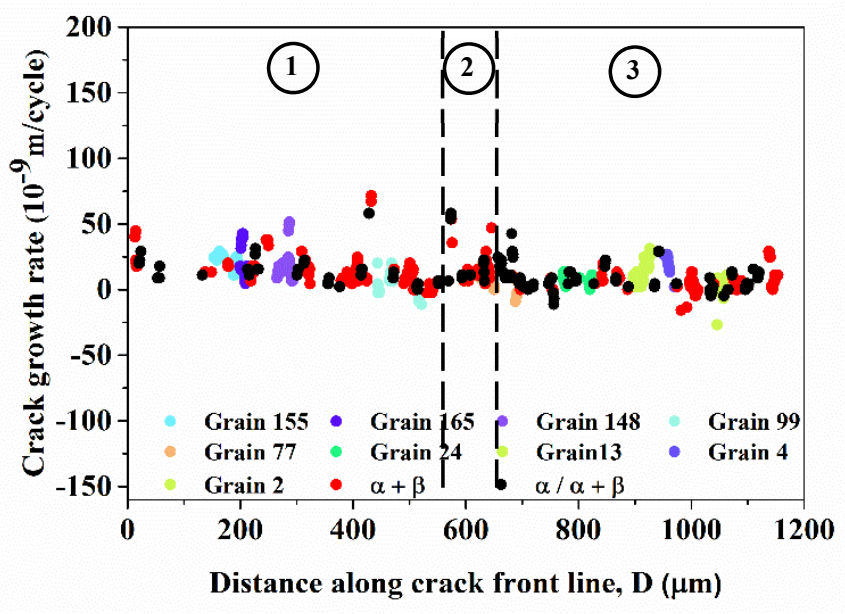

(c)

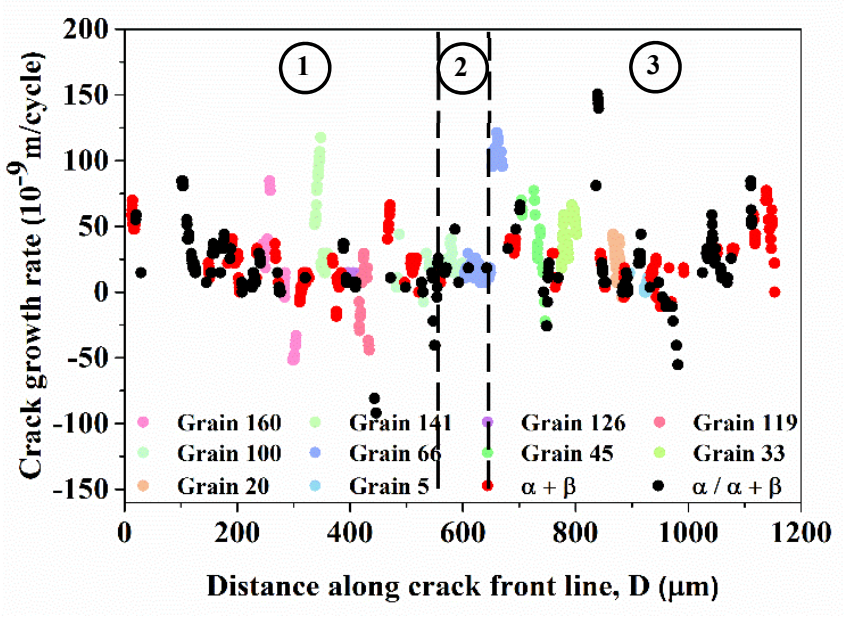

(d)

Figure 4. Spatial crack growth rate variation in the microstructure $(\alpha, \alpha+\beta$ phases and $\alpha / \alpha+\beta$ interface) at (a) 8900 , and (b) 10600 cycles for the first region, following by (c) 17230, and (d) 18560 cycles for the second region. Grain colors corresponds to the IPF map.

In order to quantify the crack front and microstructure interaction in each cycle, the temporal crack front percentage growing in $\alpha, \alpha+\beta$ phases and on the interface $(\alpha / \alpha+\beta)$ in both regions were determined. In the first region, the crack front percentage in $\alpha+\beta$ phase is close to the average microstructural fraction of $\alpha+\beta$. However, this percentage is higher and lower than the average fractions in $\alpha$ phase and interface, respectively (Figure 5(a)). Therefore, the crack front path grows at some location in the $\alpha$ phase without passing through interface. This local growth in $\alpha$ phase, depends on crack path morphology and grains crystallographic orientations, which induce an irregular crack front shape. The crack has the highest average growth rate in $\alpha$ phase (Figure 5(b)) as compared to other microstructural parts that have lower rates and vary from cycle to cycle.

In the second region, the crack front grows into the $\alpha$ phase with lower fractions than the average microstructural ones. However, the crack tends to follow the $\alpha+\beta$ phase and interface with higher rates than the microstructural fraction. This may be due to crack growth on unfavorable crystallographic orientation in $\alpha$ phase, which induces a favorable path for the crack to grow in $\alpha+\beta$ phase and interface. The crack growth rates gradually increase in the $\alpha, \alpha+\beta$ phases and interface till reaching the highest average crack growth rate (Figure 5(b)). The crack growth rate gradually increases in the $\alpha+\beta$ phase and interface with lower values as compared to $\alpha$ phase. 


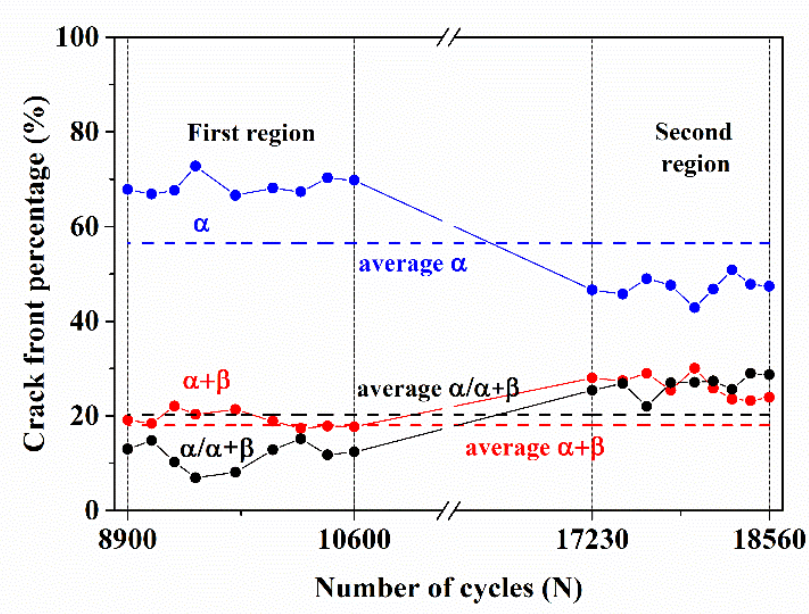

(a)

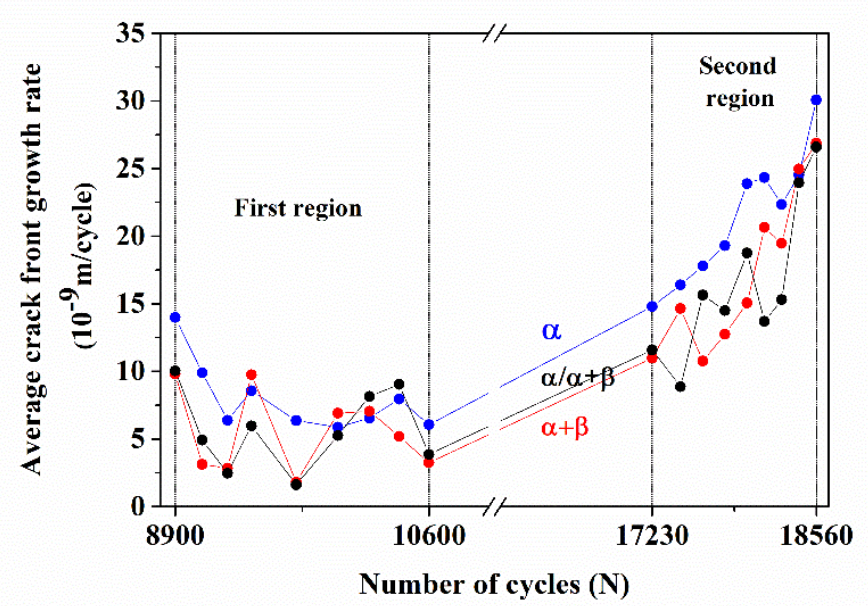

(b)

Figure 5. Temporal crack growth behavior in the microstructure $(\alpha, \alpha+\beta$ phases and $\alpha / \alpha+\beta$ interface) in the first region ( 8700 to 10600 cycles) and in the second region (17000 to 18560 cycles) characterized by (a) crack front percentage as compared to the average microstructural fraction, and (b) average crack front growth rate.

\subsection{Crack morphology}

Two distinct types of crack planes in the $\alpha$ phase are observed, which are the facet-like and non-facet-like planes. As it can be seen from Figure 6(a), crack front is growing through facet-like planes with higher percentage as compared to the non-facet ones in the beginning of the first region. However, at the end of the first region the crack front percentage in facet-like planes decreases. By comparing crack growth in each cycle, the results show that the facet-like average growth is 1.8 to 8.9 times faster than the non-facet-like growth in the first region (Figure 6(b)). This shows that crack growth in facet-like planes are prone to local rate variations due to the crack front interaction with non-facet like planes.

In the second region, crack front grows in facet-like planes with a lower percentage, however, this percentage increases at the end of second region mainly due to the formation of cracks at secondary locations as compared to the crack front. As contrary to the first region, crack grows in facet-like planes with lower average crack growth rates as compared to the non-facet like planes with ratios varying from 0.51 to 1.1 in the second region. In this region, the crack front is larger, so higher number of grains are enclosed in a larger plastic zone. As a result, cracks are formed in grains with high Schmid factors as facet-like planes at secondary locations within the plastic zone, which leads to an increase in facet-like planes. Crack growth rate at secondary locations gradually decreases until the crack stops to grow. Our results show that an increase in facet-like plane percentage does not correlate with the increase in local crack growth rate. However, the crack and grains interaction may affect this local crack growth rate and may induce some variations.

The morphology of crack with facet-like planes in grain 106 in the first region and grain 141 in the second region are shown in Figures 7(a) and (b), respectively. The crack planes are growing in an irregular morphology in the case of grain 106. The crack growth is higher in the center of the grain as compared to the surface, which shapes a triangle facet plane. The decrease in crack growth on the sides may be due to the interaction with the neighboring grains. On the other hand, the facet planes growth in grain 141 in the second region shows a more regular shape. 


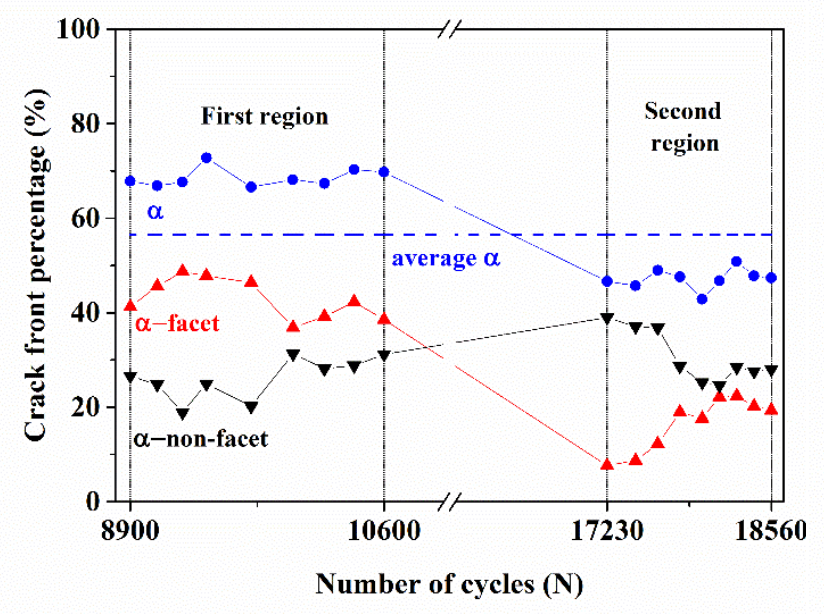

(a)

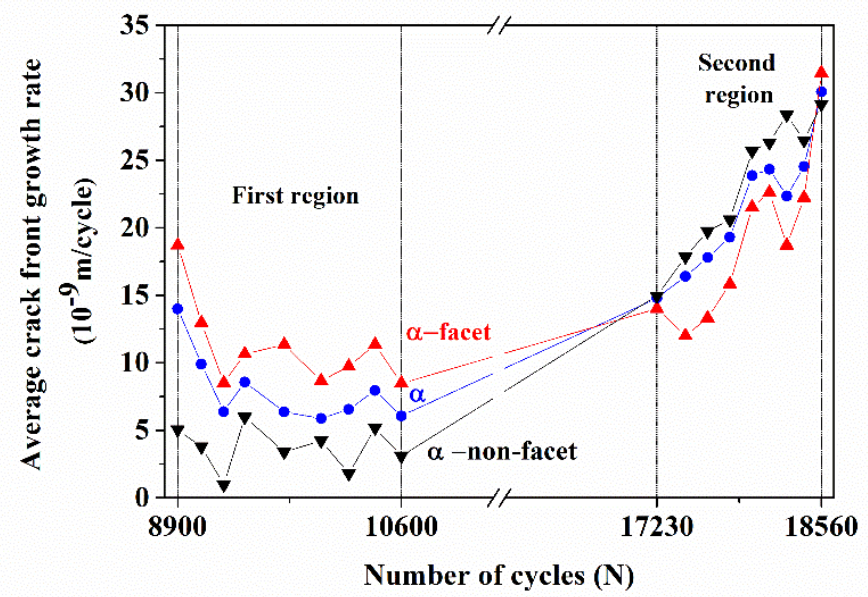

(b)

Figure 6. Crack growth behavior in the $\alpha$ phase characterized by (a) crack front percentage, (b) average crack front growth rate in the first region ( 8700 to 10600 cycles) and second region (17000 to 18560 cycles).

\section{Crack with}

Number of cycles

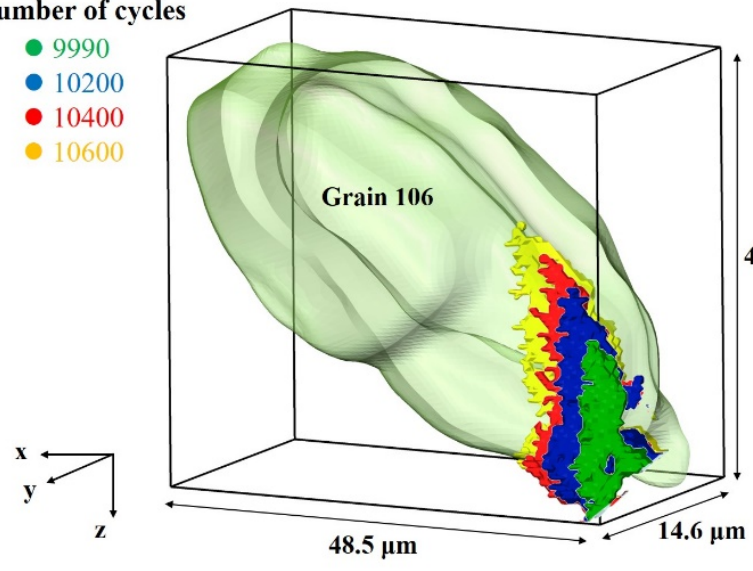

(a)

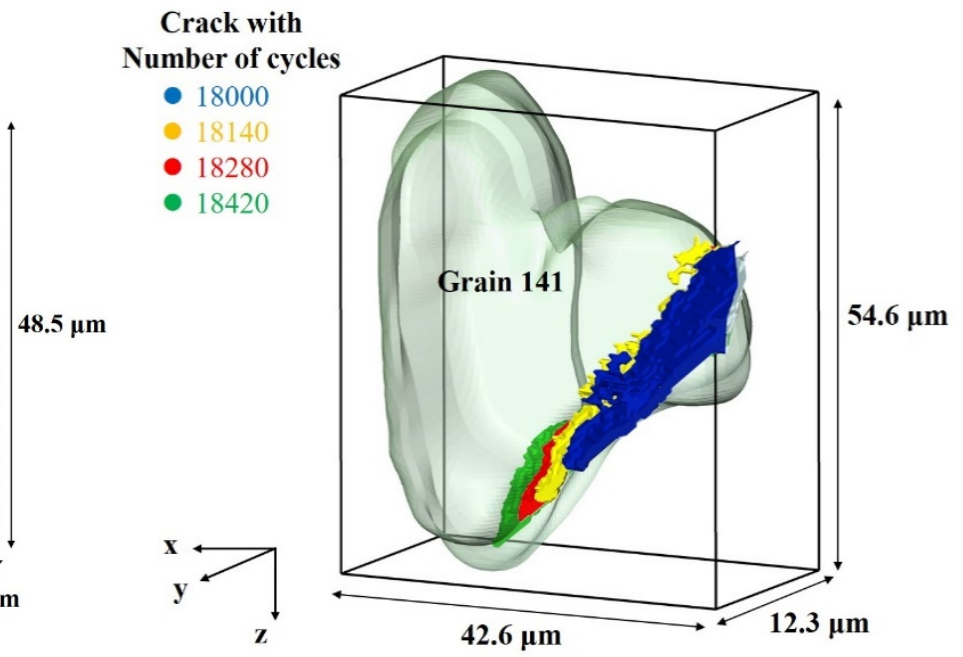

(b)

Figure 7. Superimposed images of crack with facet-like planes crack with colors corresponding to different cycles in (a) grain 106 in first region, and (b) grain 141 in the second region.

\section{Discussion}

\subsection{Transitional behavior of crack plane}

As explained in the introduction section, deviations from crystallographic planes have been reported in the previous studies $[9,21]$. In this study, crack plane deviation angle from the favorable crystallographic plane, $\psi$, (plane with the highest Schmid factor) in cracked grains in facet and non-facet-like fracture paths are shown in Figures 8 and 9. In the first region, crack grows on the basal planes in facet-like paths and on the non-basal planes (prismatic) in non-facet-like paths. Crack grows in most of the grains with relatively high Schmid factors by a maximum of 5 degrees deviation from the favorable planes in this region. These deviations may be due to the variation of crystallographic orientation of grains in EBSD sections or due to the calculation of crack plane orientation with respect to the grains plane.

Figure 8(a) shows that crack growth on a favorable plane (circled grains in Figure 8(a)) leads to a high mismatch angle with neighboring cracked grains. However, neighboring cracked grains enforce the crack plane to have a lower average mismatch 
angle, $\varphi_{\text {ave }}$, (dotted circle grains in Figure 8(a)). As a consequence, crack grows into another plane with a lower Schmid factors and with deviation from the favorable plane.

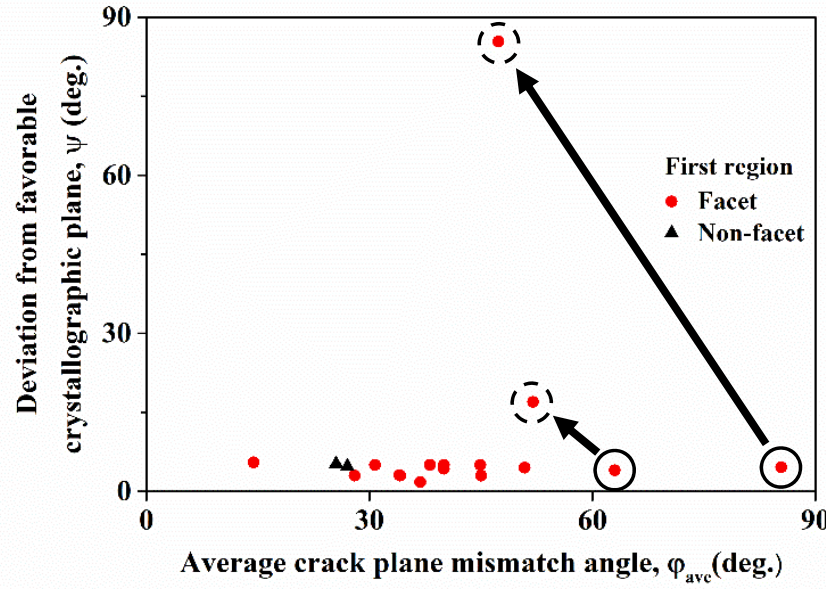

(a)

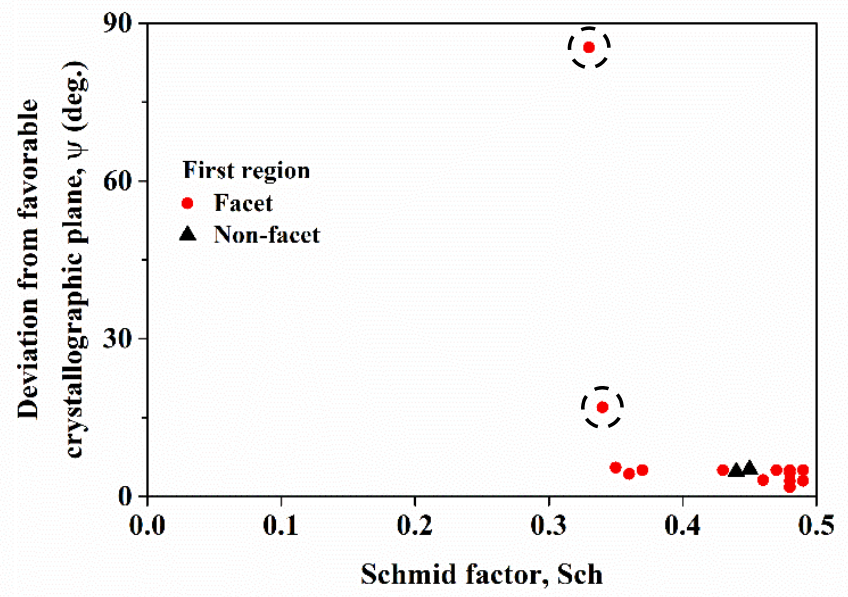

(b)

Figure 8. Deviation from favorable crystallographic plane in $\alpha$ grains in the first region with respect to a) average crack plane mismatch angle, $\varphi_{\text {ave, }}$ and (b) Schmid factor, Sch. The dotted circles show the $\alpha$ grains with lower $\varphi_{\text {ave }}$ angles and lower Schmid factors, respectively.

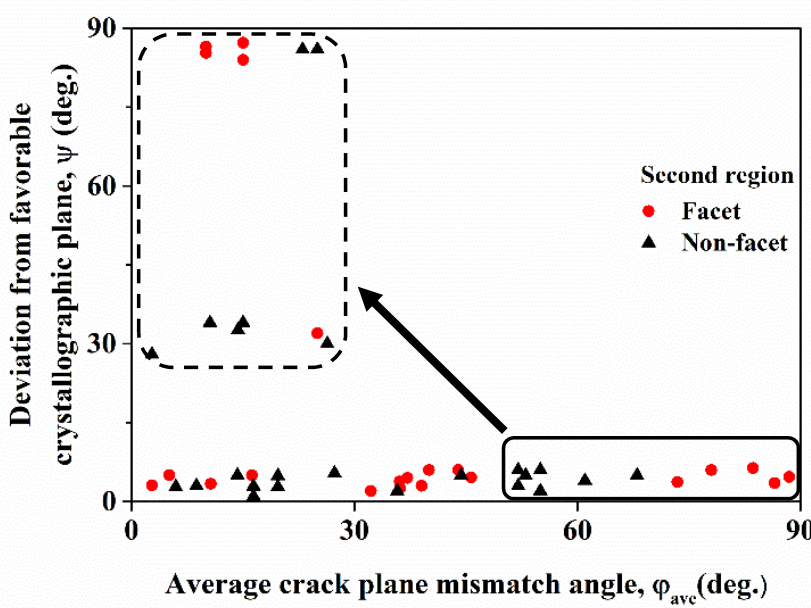

(a)

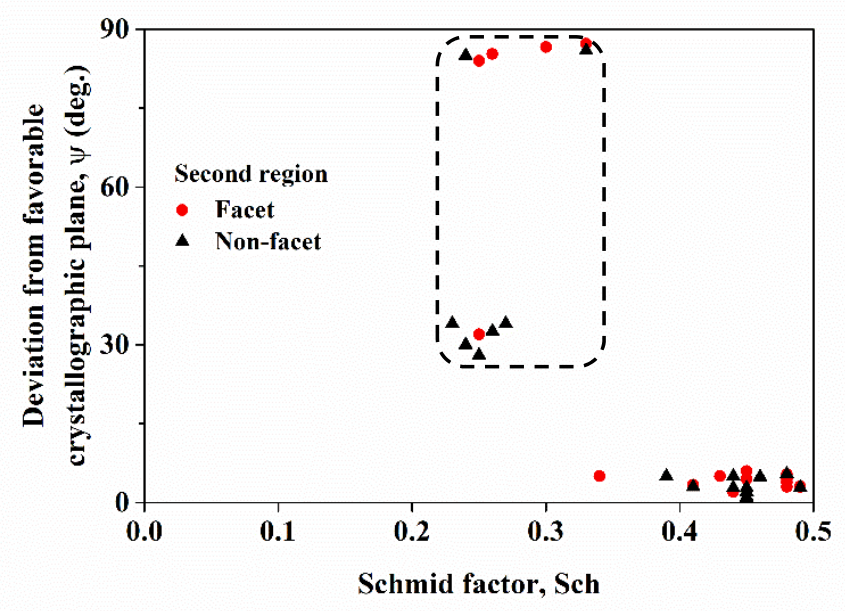

(b)

Figure 9. Deviation from favorable crystallographic plane in $\alpha$ grains in the second region with respect to a) average crack plane mismatch angle, $\varphi_{\text {ave }}$, and (b) Schmid factor, Sch. The dotted contour shows the $\alpha$ grains with lower $\varphi_{\text {ave }}$ angles and lower Schmid factors, respectively.

As the crack grows into the second region, some facet-like planes also grow on prismatic and a pyramidal plane as contrary to the first region. There is an increase in the non-facet-like crack paths and an overall increase in cracked grains with high average mismatch angles as compared to the first region (contoured grains in Figure 9(a) versus circled ones Figure 8(a)). However, the interaction with neighboring grains enforce the crack to grow in planes with lower mismatch angles (dotted contour grains in Figure 9(a)).

As the crack front grows towards the second region, the plastic zone size becomes much larger than the average grain size. This leads to an increase in number of grains that interact with the crack front. This interaction induces a decrease in the average crack plane mismatch angles in some grains which leads to high deviations from favorable crystallographic planes. These 
deviations result in crack growth on planes with lower Schmid factors (unfavorable planes) which induces a decrease in the local plasticity and the maximum shear stress on those planes (Figure 9(b)).

To elucidate the crack transitional behavior and its selection of crystallographic planes in grains, the crack plane in grain 149 and 160 is shown in Figure 10(a). The crack is growing on plane 1 with a high basal Schmid factor in a facet-like path, which is a basal plane, in grain 160. As the crack grows, there are three optional planes (numbered as 2, 3 or 4 in Figure 10(b)) that can be followed in order to grow from grain 160 into the grain 149. The growth from plane 1 into plane 2 minimizes the mismatch angles between the two possible planes as shown in Figure 10(c). Thus, the crack grows in plane 2 with the lowest Schmid factor leading to a decrease in crack growth and crack arrest in grain 149. This behavior lead to crack bifurcation and formation of secondary cracks with facet-like paths in the second region. Thus, it can be concluded that unfavorable crystallographic plane selections decrease the crack front percentage in $\alpha$ phase in the second region (see Figure 6(a)).
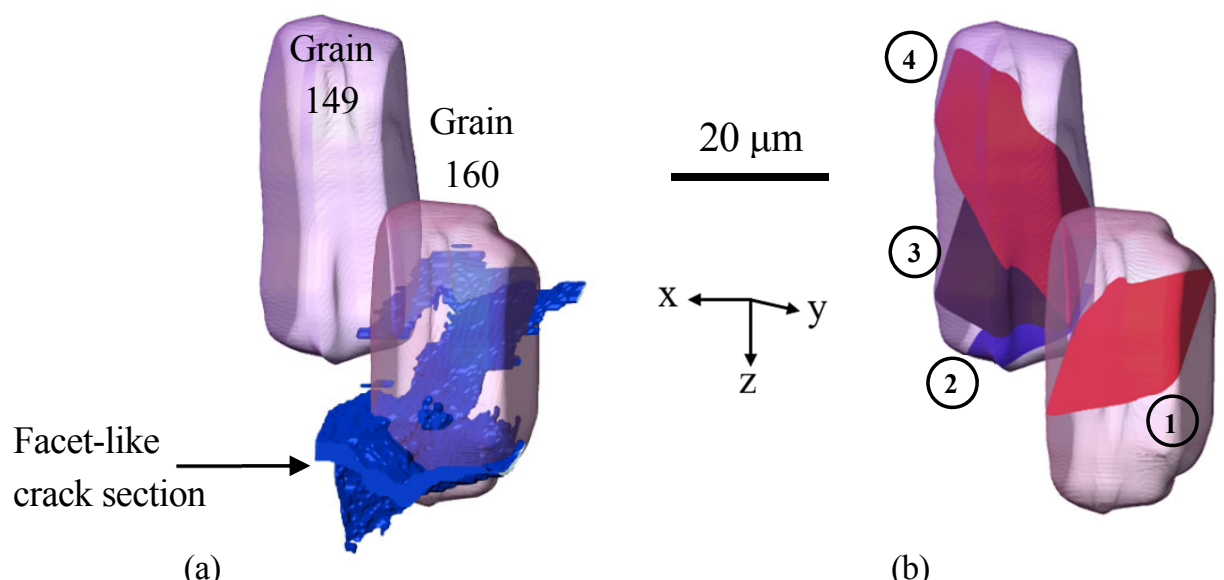

(b)

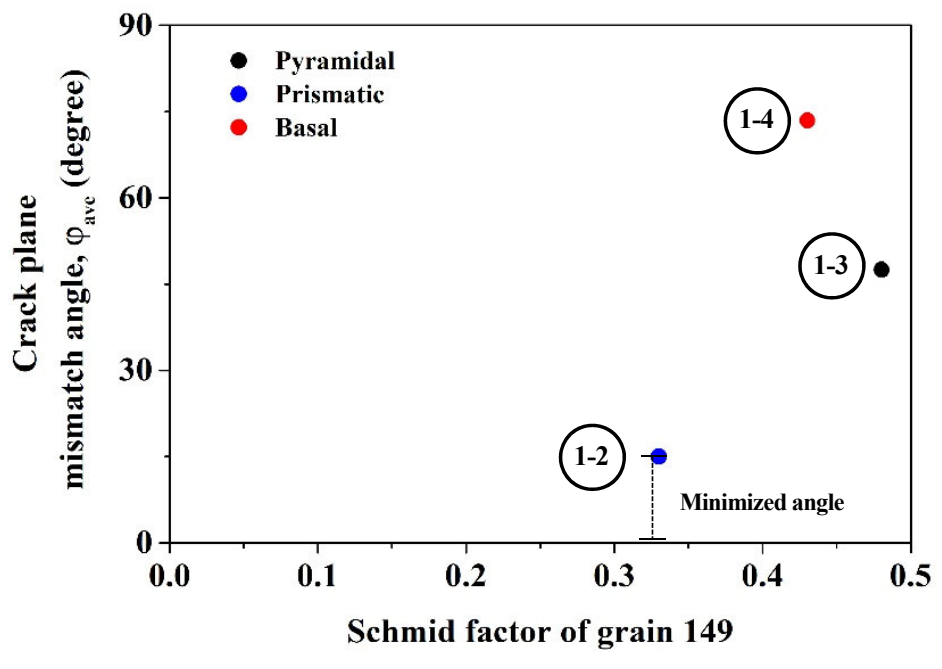

(c)

Figure 10. Crack and grains interaction, (a) facet-like crack section in grains 149 and 160, (b) crack on the basal plane 1 in grain 160 with three optional planes numbered as 2, 3, 4 corresponding to prismatic, pyramidal and basal planes, respectively in grains 149, and c) mismatch between crack plane 1 and the three others crystallographic planes as a function of Schmid

factor of grain 149 . 


\subsection{Crack and grains interaction effect on crack growth rate}

As it was explained, crack grows mainly on crystallographic planes with maximum Schmid factor and shear stress in the first region. A decrease in the average mismatch angle with neighboring cracked grains leads to a deviation from the favorable plane so crack grows on a plane with lower Schmid factor and lower shear stress which may induce a decrease in crack growth rate. In the first region, a typical effect of the aforementioned crack and grains interaction on crack growth rate is shown in Figures 11(a) and (b), respectively. Three neighboring grains are numbered as 103,106, and 93. At 9990 cycles, crack grows into grain 103 by decreasing the mismatch angle with neighboring grains (arrow towards the dotted circle grain in Figure 11(a)) which induces a high deviation from the favorable crystallographic plane. As a consequence, crack grows on basal plane with a Schmid factor of 0.33 (unfavorable plane) as compared to the prismatic plane with a Schmid factor of 0.43 (favorable plane), so there is a lower local plasticity on the basal plane. This low local shear stress and high mismatch angle may cause the low crack growth rate in this grain. At the same cycle, crack starts to grow in grain 106 with a similar mismatch angle but close to the favorable plane (basal plane), which leads to high crack growth rates. At 10200 cycles, crack grows in grain 93 with a deviation from the basal plane in order to decrease its mismatch angle (arrow towards the dotted circle grain in Figure 11(a)), which lead to low crack growth rates. The low crack growth rates in grains 103 and 93, induce a gradual decrease in crack growth rate of grain 106 (Figure 11(b)).

In the second region, a typical effect of facet-like crack and grains interaction is shown in Figures 12(a) and (b). Three neighboring grains are numbered as 160,149 and 141. The crack in grain 141 follows the other grains on the crack front line, so it is considered as the main crack; however, at 17640 cycles crack bifurcates and grows into grain 160 with a high Schmid factor (Figure 12(a)) which leads to the higher crack growth rate in this grain (Figure 12(b)). At 17820 cycles, crack starts to grow from grain 160 into the neighboring grain 149; as a consequence, the crack growth rates in both grains start to decrease until 18140 cycles. The crack plane grows in grain 149 with a high deviation from the favorable plane in order to have a lower mismatch angle (arrow towards the dotted circle in Figure 12(a)). This leads to a growth on a plane with a lower Schmid factor as compared to the favorable plane ( 0.33 versus 0.43 ), so the crack growth rate in grain 149 starts to decrease and induces a decrease in crack growth rate of grain 160. As a result of a decrease in crack growth rate in the secondary path section (grain 149 and 160), the crack growth rate in the main crack in grain 141 gradually increases. 

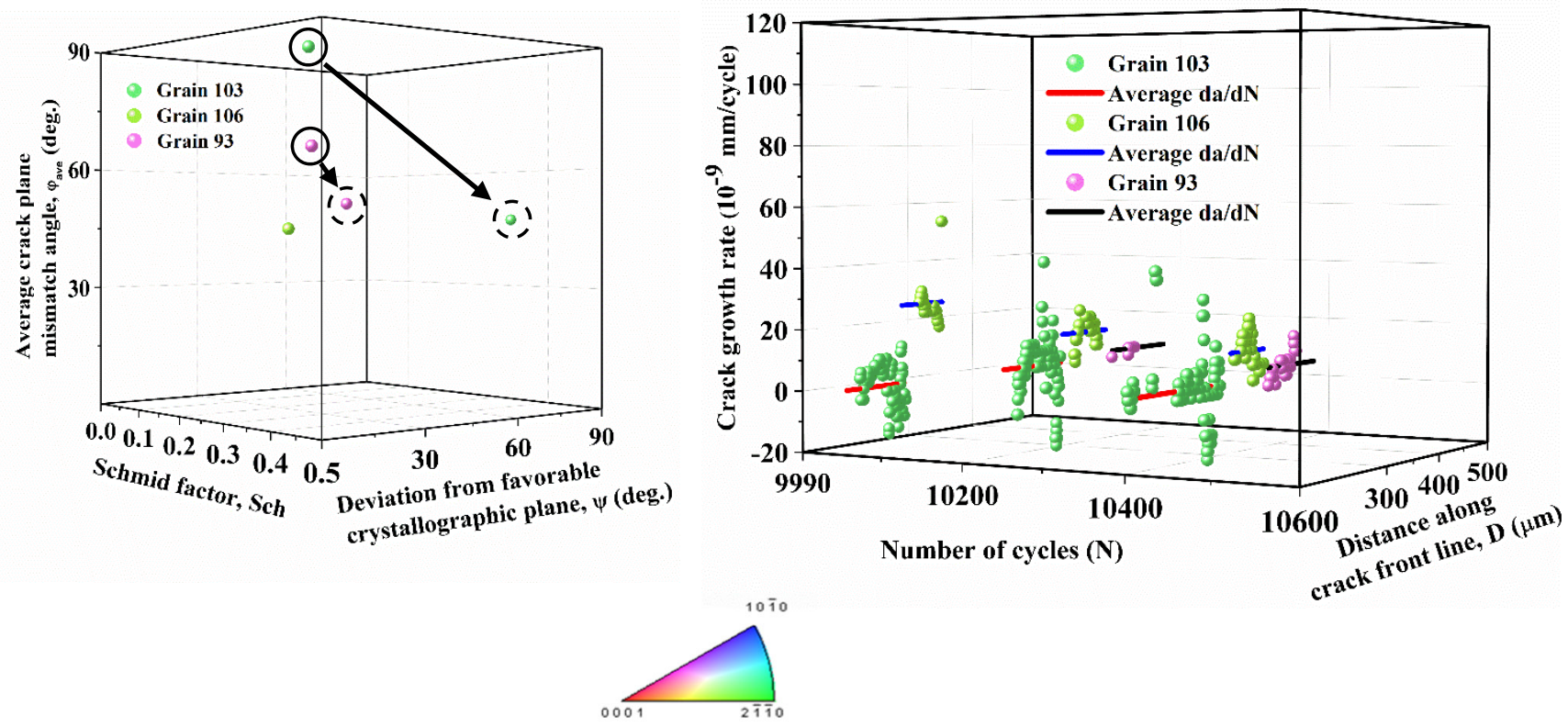

(a)

(b)

Figure 11. Crack and three neighboring grains interaction described by, (a) Schmid factor, crack plane deviation from favorable crystallographic plane and average crack plane mismatch angle with neighboring grains for the three grains, b) decrease in average crack growth rate in grain 106 due to the interaction with the neighboring grains 103 and 93 . Colors of grains corresponds to the IPF map.
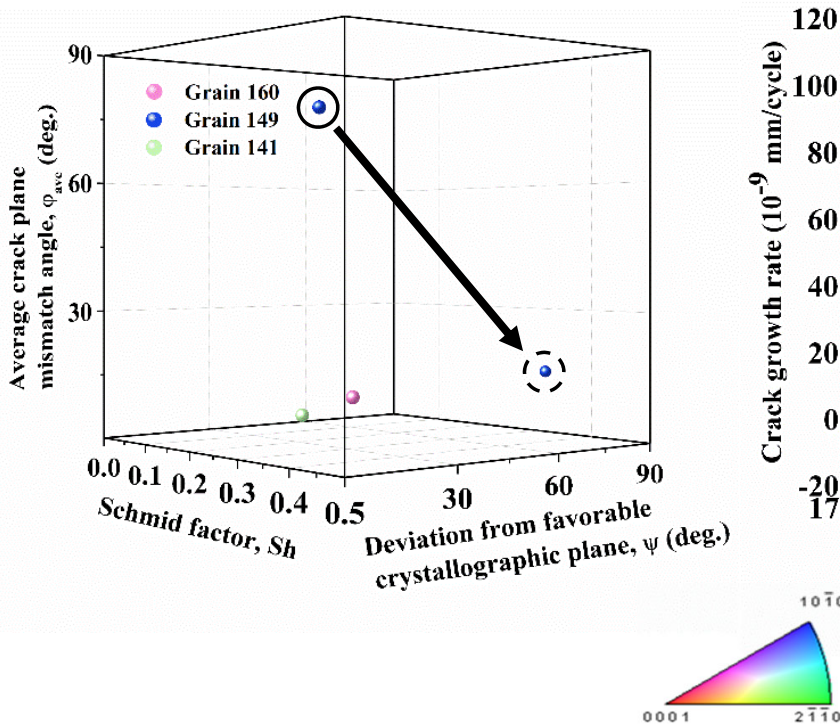

(a)

Figure 12. Crack and three neighboring grains interaction described by, (a) Schmid factor, crack plane deviation from favorable crystallographic plane and average crack plane mismatch angle with neighboring grains, (b) decrease in average crack growth rate of grain 160 due to interaction with grains 149 and 141 . Colors of grains corresponds to the IPF map. 


\section{Conclusions}

The effects of 3D microstructure on the short crack growth rates in a bimodal Ti-6Al-4V alloy is investigated at two crack front regions. In situ computed tomography to measure short crack growth in the $\alpha, \alpha+\beta$ phases and interface. The crystallographic orientations of 3D grains in the $\alpha$ phase was identified using serial sectioning coupled with electron backscattering diffraction (EBSD).

In the first region, crack front preferably grows in $\alpha$ phase higher than the average microstructural fraction, but it grows into $\alpha / \alpha+\beta$ (interface) and $\alpha+\beta$ phase below and close to average fraction, respectively. Crack growth rate in $\alpha$ phase has the highest crack growth rate as compared to $\alpha+\beta$ phase and interface. This may be due to the crack growth on the facet-like planes with higher average growth rates than the non-facet-like ones in the first region. However, it was shown that the local crack growth rates in facet-like or non-facet-like planes depends on the interaction with neighboring cracked grains at the crack front.

As the short crack front grows in the second region, crack front percentage following the $\alpha$ phase decreases below the average microstructural fraction but the crack percentage following the $\alpha+\beta$ phase and interface increase. The average crack growth rates gradually increase in $\alpha, \alpha+\beta$ phases and interface. There is a decrease in the percentage of crack front growing in facetlike planes but this percentage increases at the end of the second region due to crack bifurcations and due to formation of secondary cracks.

The crack plane grows mainly on the crystallographic planes with maximum Schmid factor and shear stress (favorable planes) in the first region. The interaction between the 3D crack and grains induces a decrease in the average crack plane mismatch angle with neighboring cracked grains, which leads to crack plane deviation from the grain's favorable plane (maximum Schmid factor). As a result, crack grows in a plane with lower Schmid factor and shear stress. In the second region, there is an increase in the number of grains enclosed in the plastic zone which interact with the crack front. As a result, crack tends to minimize its plane angle with neighboring cracked grain leading to an increase in number of grains with deviations from the favorable planes. It can be concluded that the crack and grain interaction is the main factor determining the crack plane mismatch angle and deviation from favorable planes, which causes variations in local crack growth rates.

\section{Acknowledgments}

The synchrotron radiation experiments were performed at SPring-8 with the approval of Japan Synchrotron Radiation Research Institute (JASRI) through proposal number of 2016A0076 and 2016B0076. This work was supported through the grant-in-aid for scientific research from Structural Materials for Innovation (SM4I) of the Cross-ministerial Strategic Innovation Promotion Program (SIP) and light metal educational foundation (LMEF).

\section{References}

[1] C.M. Ward-Close, C.J. Beevers, The influence of grain orientation on the mode and rate of fatigue crack growth in $\alpha$ titanium, Metall. Mater. Trans. A. 11 (1980) 1007-1017.

[2] D. Banerjee, J.C. Williams, Perspectives on titanium science and technology, Acta Mater. 61 (2013) 844-879.

[3] F.P.E. Dunne, A. Walker, D. Rugg, A systematic study of hcp crystal orientation and morphology effects in polycrystal deformation and fatigue, in: Proc. R. Soc. London A Math. Phys. Eng. Sci., The Royal Society, 2007: pp. 1467-1489.

[4] Z. Zhang, M.A. Cuddihy, F.P.E. Dunne, On rate-dependent polycrystal deformation: the temperature sensitivity of cold dwell fatigue, in: Proc. R. Soc. A, The Royal Society, 2015: p. 20150214.

[5] T. Sakai, M.E. Fine, Basal slip of Ti-Al single crystals, Scr. Metall. 8 (1974) 545-547. 
[6] I.P. Jones, W.B. Hutchinson, Stress-state dependence of slip in Titanium-6Al-4V and other HCP metals, Acta Metall. 29 (1981) 951-968.

[7] F. Bridier, P. Villechaise, J. Mendez, Analysis of the different slip systems activated by tension in a $\alpha / \beta$ titanium alloy in relation with local crystallographic orientation, Acta Mater. 53 (2005) 555-567.

[8] F. Bridier, P. Villechaise, J. Mendez, Slip and fatigue crack formation processes in an $\alpha / \beta$ titanium alloy in relation to crystallographic texture on different scales, Acta Mater. 56 (2008) 3951-3962.

[9] I. Bantounas, D. Dye, T.C. Lindley, The effect of grain orientation on fracture morphology during high-cycle fatigue of Ti-6Al-4V, Acta Mater. 57 (2009) 3584-3595.

[10] V. Sinha, M.J. Mills, J.C. Williams, J.E. Spowart, Observations on the faceted initiation site in the dwell-fatigue tested Ti-6242 alloy: crystallographic orientation and size effects, Metall. Mater. Trans. A. 37 (2006) 1507-1518.

[11] S. Birosca, J.Y. Buffiere, M. Karadge, M. Preuss, 3-D observations of short fatigue crack interaction with la2mellar and duplex microstructures in a two-phase titanium alloy, Acta Mater. 59 (2011) 1510-1522.

[12] E.E. Sackett, L. Germain, M.R. Bache, Crystal plasticity, fatigue crack initiation and fatigue performance of advanced titanium alloys, Int. J. Fatigue. 29 (2007) 2015-2021.

[13] F. McBagonluri, E. Akpan, C. Mercer, W. Shen, W.O. Soboyejo, An investigation of the effects of microstructure on dwell fatigue crack growth in Ti-6242, Mater. Sci. Eng. A. 405 (2005) 111-134.

[14] X. Demulsant, J. Mendez, Influence of environment on low cycle fatigue damage in Ti6Al4V and Ti 6246 titanium alloys, Mater. Sci. Eng. A. 219 (1996) 202-211.

[15] K. Tanaka, Y. Akiniwa, Propagation and non-propagation of small fatigue cracks, in: ICF7, Houst. $1989,1989$.

[16] A.L. Pilchak, R.E.A. Williams, J.C. Williams, Crystallography of fatigue crack initiation and growth in fully lamellar Ti-6Al-4V, Metall. Mater. Trans. A. 41 (2010) 106.

[17] G.M. Castelluccio, W.D. Musinski, D.L. McDowell, Computational micromechanics of fatigue of microstructures in the HCF-VHCF regimes, Int. J. Fatigue. 93 (2016) 387-396.

[18] A. Fatemi, D.F. Socie, A critical plane approach to multiaxial fatigue damage including out of phase loading, Fatigue Fract. Eng. Mater. Struct. 11 (1988) 149-165.

[19] X. Chen, S. Xu, D. Huang, A critical plane strain energy density criterion for multiaxial low cycle fatigue life under non proportional loading, Fatigue Fract. Eng. Mater. Struct. 22 (1999) 679-686.

[20] I. Bantounas, T.C. Lindley, D. Rugg, D. Dye, Effect of microtexture on fatigue cracking in Ti-6Al-4V, Acta Mater. 55 (2007) 5655-5665.

[21] A.L. Pilchak, Fatigue crack growth rates in alpha titanium: faceted vs. striation growth, Scr. Mater. 68 (2013) 277280 .

[22] T. Zhai, A.J. Wilkinson, J.W. Martin, A crystallographic mechanism for fatigue crack propagation through grain boundaries.pdf, 48 (2000) 4917-4927.

[23] T. Zhai, X.P. Jiang, J.X. Li, M.D. Garratt, G.H. Bray, The grain boundary geometry for optimum resistance to growth of short fatigue cracks in high strength Al-alloys, Int. J. Fatigue. 27 (2005) 1202-1209. doi:10.1016/j.ijfatigue.2005.06.021.

[24] A.W. Sommer, D. Eylon, On fatigue crack Propagation of Titanium Alloys under Dwell Time Conditions, Metall. Trans. A. 14 (1982) 2178-2181.

[25] H. Toda, A. Takijiri, M. Azuma, S. Yabu, K. Hayashi, D. Seo, M. Kobayashi, K. Hirayama, A. Takeuchi, K. Uesugi, Damage micromechanisms in dual-phase steel investigated with combined phase-and absorption-contrast tomography, Acta Mater. 126 (2017) 401-412.

[26] H. Toda, I. Sinclair, J.Y. Buffiére, E. Maire, T. Connolley, M. Joyce, K.H. Khor, P. Gregson, Assessment of the fatigue crack closure phenomenon in damage-tolerant aluminium alloy by in-situ high-resolution synchrotron X-ray microtomography, Philos. Mag. 83 (2003) 2429-2448. doi:10.1080/1478643031000115754. 
[27] A. Burvall, U. Lundström, P.A.C. Takman, D.H. Larsson, H.M. Hertz, Phase retrieval in X-ray phase-contrast imaging suitable for tomography, Opt. Express. 19 (2011) 10359-10376.

[28] C.G. Schroer, P. Cloetens, M. Rivers, A. Snigirev, A. Takeuchi, W. Yun, High-resolution 3D imaging microscopy using hard X-rays, Mrs Bull. 29 (2004) 157-165.

[29] D. Paganin, S.C. Mayo, T.E. Gureyev, P.R. Miller, S.W. Wilkins, Simultaneous phase and amplitude extraction from a single defocused image of a homogeneous object, J. Microsc. 206 (2002) 33-40. 